\title{
Predictions for Upscaling Sonoluminescence
}

\author{
Sascha Hilgenfeldt ${ }^{1}$ and Detlef Lohse ${ }^{2}$ \\ ${ }^{1}$ Division of Engineering and Applied Sciences, Harvard University, 29 Oxford Street, Cambridge, Massachusetts 02138 \\ ${ }^{2}$ Department of Applied Physics and J.M. Burgers Centre for Fluid Mechanics, University of Twente, P.O. Box 217, \\ 7500 AE Enschede, The Netherlands
}

(Received 14 November 1997; revised manuscript received 5 November 1998)

\begin{abstract}
Only a small fraction of the high-dimensional parameter space that governs the occurrence of stable single-bubble sonoluminescence (SBSL) has been explored so far. We predict that decreasing the acoustic driving frequency $f$ upscales SBSL. More specifically, at $f=5 \mathrm{kHz}$ we expect more than 100 times as many photons per flash as at $f=20 \mathrm{kHz}$ and a flash width of about $1000 \mathrm{ps}$. The application of lower frequencies has to be assisted by reducing the partial inert gas pressure of the dissolved gas (e.g., stronger degassing) to maintain diffusive stability of the bubbles. [S0031-9007(98)08344-6]
\end{abstract}

PACS numbers: 78.60.Mq, 43.25. $+\mathrm{y}, 47.55 . \mathrm{Dz}$

The astonishing energy focusing capabilities of a bubble in single-bubble sonoluminescence (SBSL) [1,2] have intrigued many researchers and triggered numerous experimental [2,3] and theoretical [4] studies aimed at determining the central temperatures and emitted light intensity of SBSL bubbles. Most experiments and theories indicate that the temperatures (or equivalent energy densities) inside the bubble can reach at least several $10^{4} \mathrm{~K}$ and the short (80-300 ps [5,6]) light pulses consist of up to $10^{7}$ photons in the visible range [2]. The question now arises if the experimental parameters can be altered such that these figures can be pushed to even more extreme values, i.e., can SBSL be upscaled?

Experimentally [2] it was found that an efficient way to achieve more intense light is to cool the water. Decreasing the water temperature from 33 to $2.5^{\circ} \mathrm{C}$ gives nearly 1000 times more photons per pulse. Following the hydrodynamical/chemical approach to SBSL [7-10], the water temperature dependence was quantitatively accounted for by considering the temperature dependence of the material constants of water [11].

In this paper we focus on the upscaling of SBSL by reducing the driving frequency $f$, a procedure suggested by Apfel [12]. The effect of reducing the frequency is twofold: (i) In the SL regime the dynamics of the bubble radius $R(t)$ is characterized by a long and relatively slow expansion that occurs during the negative pressure phase of the driving, and by a subsequent violent collapse. Because a smaller frequency gives the bubble more time to expand, it leads to a larger expansion ratio $R_{\max } / R_{0}$ (maximum radius divided by ambient radius) and a stronger collapse. Indeed, the example of Fig. 1 shows that, in comparison to frequencies commonly used today in experiment (e.g., $f \approx 20-40 \mathrm{kHz}$ ), more violent collapses can be reached (at fixed $R_{0}$ ) with smaller $f$. (ii) For smaller $f$ the threshold of shape instability which limits the SBSL regime [8] is shifted towards larger bubbles which potentially emit more sonoluminescence light. Indeed, experiments with smaller $f$ by Barber and Putterman [13] and by Cordry [14] found brighter SL bubbles. But what is the maximum light intensity which can be expected, and how should one choose the forcing pressure $P_{a}$ and the gas concentration in the liquid to achieve an optimal photon yield? The present study will make quantitative predictions for answers to these questions.

Our analysis of upscaled SBSL is based on the hydrodynamical approach to SBSL [7-10], augmented by an approximate description of the thermal bremsstrahlung of the partially ionized gas inside the bubble $[15,16]$.

The bubble is driven by the harmonic driving $P(t)=$ $-P_{a} \cos 2 \pi f t$; it responds according to the RayleighPlesset (RP) dynamics $[2,8,17,18]$. The pressure $p(R(t))$ inside the bubble is approximated by a spatially homogeneous van der Waals pressure. This approximation is well

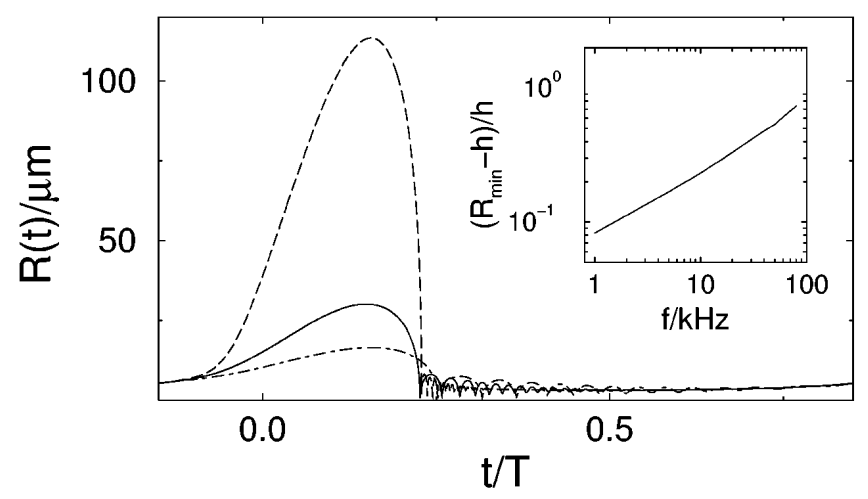

FIG. 1. Time series $R(t)$ from the RP equation for $f=5 \mathrm{kHz}$ (dashed line), $20 \mathrm{kHz}$ (solid line), and $50 \mathrm{kHz}$ (dot-dashed line) for the driving $P(t)$. The time axis is normalized with the driving period $T=1 / f$. While in all cases, $R_{0}=4 \mu \mathrm{m}$ and $P_{a}=1.2 \mathrm{~atm}$ are the same, the maximum radius $R_{\max }$ reached for $5 \mathrm{kHz}$ is almost 7 times larger than for $50 \mathrm{kHz}$, leading to a more violent collapse. This is demonstrated in the inset which shows how close the minimal radius $R_{\min }$ approaches the van der Waals hard core radius $h$ as a function of the forcing frequency $f$. 
justified for inert gases $[19,20]$ which are the only relevant gas species for stable SBSL as all molecular compounds (e.g., $\mathrm{N}_{2}, \mathrm{O}_{2}$ ) dissociate and their reaction products dissolve in water [10]. As an example we take argon and describe its concentration in the liquid by the ratio of its partial pressure $p_{\infty}^{\mathrm{Ar}}$ to the ambient pressure $P_{0}=1 \mathrm{~atm}$. The gas temperature follows from a polytropic law [16] based on results by Prosperetti [21]. The material constants are those for argon dissolved in water at $20{ }^{\circ} \mathrm{C}$. We supplement the RP equation by a corresponding ordinary differential equation (ODE) for nonspherical distortions of the bubble surface derived in [22]. This equation can be solved with a boundary layer approximation $[7,8,23]$; see [8] for details. This approximation slightly overestimates the shape instabilities due to the simplified treatment of thermal losses $[24,25]$; however, the quantitative agreement is still satisfactory [25,26].

Of the different types of shape instabilities found in Ref. [8] we focus here on the long time scale parametric instability, where the perturbations of the spherical surface can grow from cycle to cycle and finally overwhelm the bubble. At a given $f$, bubbles with ambient radii $R_{0}<R_{0}^{\max }$ are stable, with $R_{0}^{\max }$ only weakly dependent on $P_{a}$ in the SBSL regime. Numerically, we find that $R_{0}^{\max }$ increases with decreasing $f$; see Figs. 2 and 3. This finding can be accounted for by the following argument: The shape instability is driven by a parametric instability [8] on the time scale of the afterbounces, which is close to the inverse of the bubble's eigenfrequency [17] $f_{e}=$ $\sqrt{3 P_{0} / \rho_{l}} / 2 \pi R_{0}$, where $\rho_{l}$ is the liquid density. The exponential growth with time constant $f_{e}^{-1}$ is stopped after a time $\approx R_{0}^{2} / 2 \nu_{l}$, due to the damping of the $R(t)$ dynamics by the liquid viscosity $\nu_{l}$. Over the remaining afterbounce interval whose length is $\approx 1 / 2 f$ [9], the shape instabilities are themselves damped by viscosity, typically with half the time constant of the $R(t)$ damping [8].

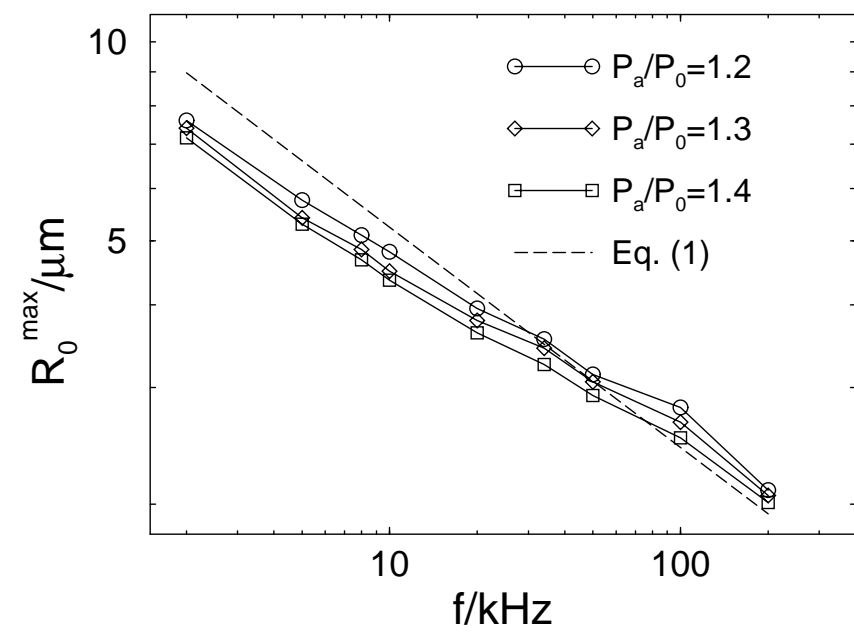

FIG. 2. Largest ambient radius $R_{0}^{\max }$ for stable argon SBSL bubbles in water at given $P_{a}=1.2,1.3$, and $1.4 \mathrm{~atm}$. The dashed line shows the estimate (1).
Putting all these facts together finally yields

$$
R_{0}^{\max } \sim\left(\frac{\rho}{3 P_{0}}\right)^{1 / 6}\left(\frac{8 \pi \nu_{l}^{2}}{f}\right)^{1 / 3}
$$

for the maximum ambient radius for which bubbles are still shape stable in the large forcing SBSL regime, in decent agreement with the numerical results of Fig. 2.

The instabilities of the Rayleigh-Taylor (RT) type have not been included here, as for small $f$ and very intense collapses they act on too short time scales as to be described within a uniform bubble model. A correct description probably requires a detailed modeling of the pressure and density variations of the bubble interior. Also, the aforementioned neglect of heat losses affects the RT instability more severely at the lower $f$. Qualitatively, the RT instability sets an upper limit to the driving pressure (cf. [8]), with this limit decreasing for smaller $f$.
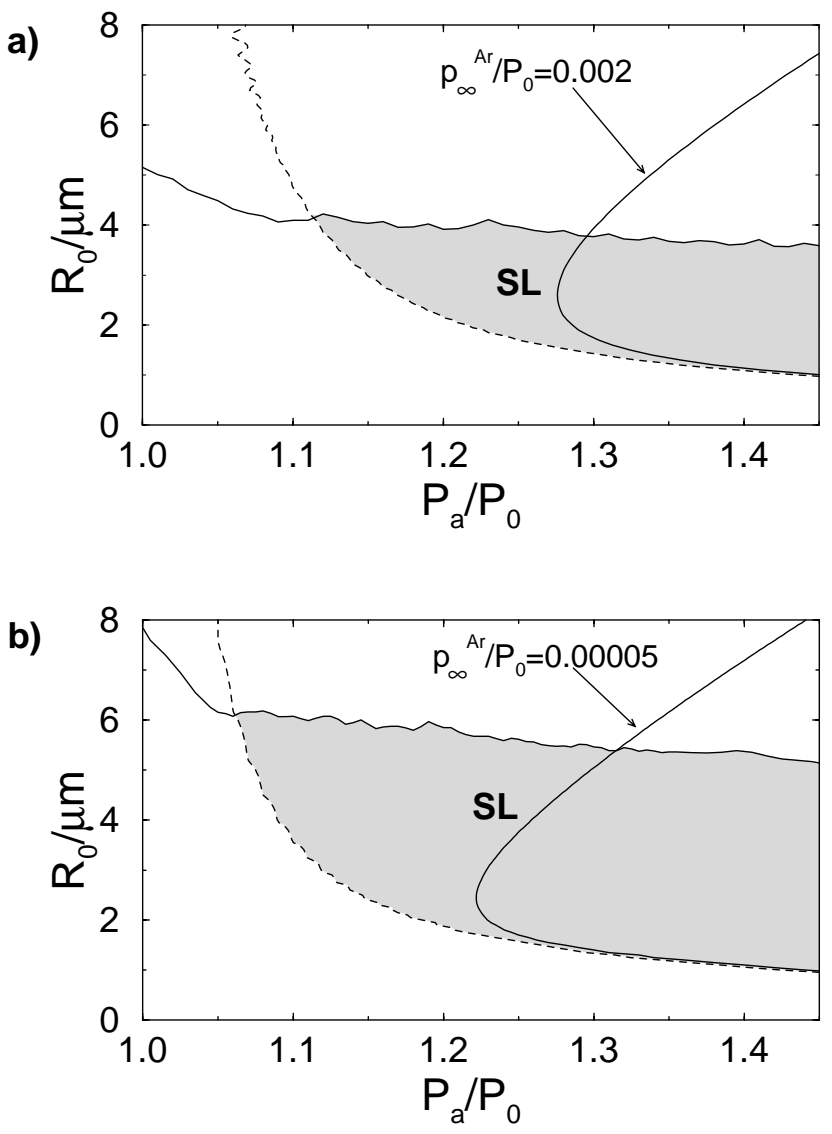

FIG. 3. Phase diagram in the $R_{0}-P_{a}$ parameter space for (a) $f=20 \mathrm{kHz}$ and (b) $f=5 \mathrm{kHz}$. The dashed lines indicate the $M_{g}=1$ Mach criterion. The thin solid lines give the threshold for parametric instability. SL bubbles fulfill both criteria (shaded region). The thick lines show the diffusive equilibria for the partial pressure of noble gas $p_{\infty}^{\mathrm{Ar}} / P_{0}$ indicated in the graph (branches with positive slope represent stable equilibria). Note that in (b) $p_{\infty}^{\mathrm{Ar}} / P_{0}$ has been chosen a factor of 40 lower than in (a) in order to achieve stable SL at high driving pressure. For the same gas concentration as in (a), the $5 \mathrm{kHz}$ driven bubble in (b) could not be stable for $P_{a} / P_{0}>1.13$. 
Sonoluminescing bubbles must also fulfill the energy focusing condition, which can be estimated by requiring $M_{g}=|\dot{R}| / c_{g} \gtrsim 1[2,7,8] ; M_{g}$ is the Mach number with respect to the speed of sound in the gas $c_{g}$. The $M_{g}=1$ curve is also given in Fig. 3; it undergoes a slight shift towards smaller $P_{a}$ and $R_{0}$ when lowering $f$ because of the enhanced collapse strength.

To summarize, because of the enhancement of shape stability and collapse strength, the SBSL regime where both the energy focusing condition and shape stability are fulfilled (shaded region in Fig. 3) is enlarged for smaller $f$, allowing for larger bubbles and stronger collapses.

Next we discuss the diffusive stability of the bubbles $[2,8,17,27,28]$. Up to now we have implicitly assumed that the ambient radius $R_{0}$ can be changed directly in experiment. However, it can only be altered indirectly by adjusting the gas concentration $[5,8,29]$. Here we are interested in diffusively stable bubbles, as they exhibit the brightest SBSL; unstable bubbles suffer from diffusive growth and shedding of microbubbles; they are less long lived and less bright $[2,26]$.

The diffusive equilibria in the SBSL regime are characterized by the condition $[8,28]$,

$$
p_{\infty}^{\mathrm{Ar}} / P_{0}=\langle p(R, t)\rangle_{4} / P_{0},
$$

where $\langle p(R(t))\rangle_{4}=\int_{0}^{T} p(R(t)) R^{4} d t / \int_{0}^{T} R^{4} d t \quad$ is a weighted mean of the gas pressure inside the bubble. The right-hand side of Eq. (2) can easily be calculated from the RP dynamics. To very good approximation $\langle p(R, t)\rangle_{4} / P_{0} \approx\left(R_{0} / R_{\max }\right)^{3} \quad[2,9] . \quad$ As $R_{\max } / R_{0}$ gets very large for the strong collapses at low $f$ (Fig. 1), the (inert) gas concentration $p_{\infty}^{\mathrm{Ar}} / P_{0}$ in the liquid must be very small for (2) to be fulfilled. Therefore, much stronger degassing is necessary to get stable bubbles at high driving pressures. In Fig. 3 we plot diffusive equilibria resulting from Eq. (2); only those with positive slope are stable. For $f=5 \mathrm{kHz}$ (Fig. 3b) the required inert gas concentration to have stable bubbles at, say, $P_{a} / P_{0}=1.3$ is well below $p_{\infty}^{\mathrm{Ar}} / P_{0}=0.01 \%$. From an experimental point of view a controlled degassing to such tiny concentrations may be difficult. Therefore we recommend to make use of argon rectification by mixing a small amount of argon with pure nitrogen, which is burned off, thus adjusting $p_{\infty}^{\mathrm{Ar}}$ to the desired value.

For the experimenter it is convenient to have predictions for phase diagrams in the space of the directly adjustable parameters $p_{\infty}^{\mathrm{Ar}} / P_{0}$ and $P_{a} / P_{0}$. These diagrams can easily be extracted from the graphs in $R_{0}-P_{a}$ space (Fig. 3). The results for three different frequencies are shown in Fig. 4. From this figure it can again be seen that lower $f$ requires lower inert gas concentrations $p_{\infty}^{\mathrm{Ar}} / P_{0}$.

The larger bubbles and the stronger collapses made us expect more light using smaller frequencies. But how much more? We can quantify the light emission using an extension of the hydrodynamic theory [16]. In

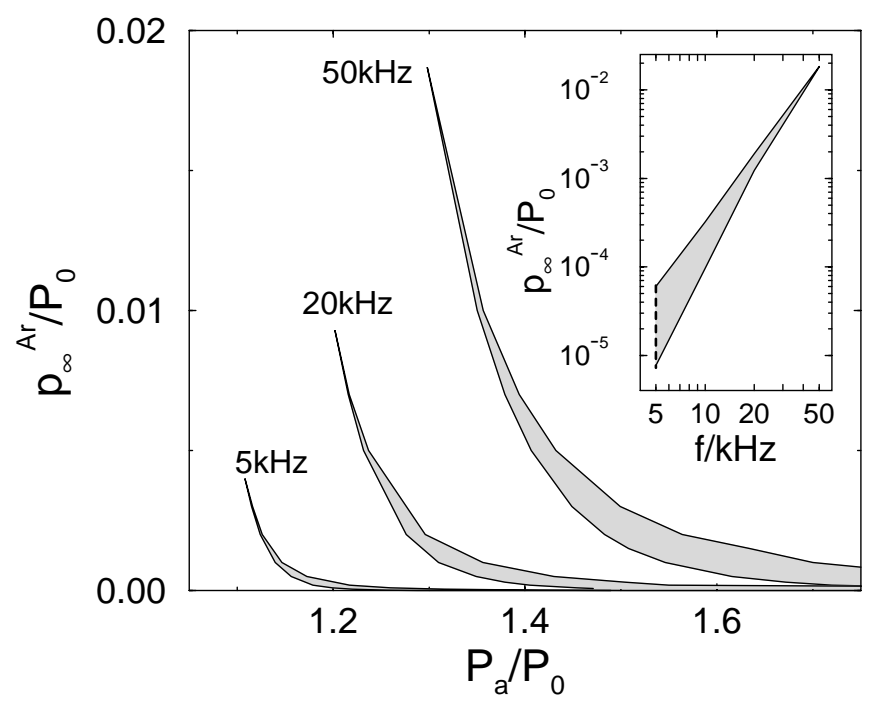

FIG. 4. Phase diagrams in the $\left(p_{\infty}^{\mathrm{Ar}} / P_{0}\right)-\left(P_{a} / P_{0}\right)$ parameter space for $f=5,20$, and 50. Stable SBSL is possible only in the small, shaded regions. For each frequency, the area below the stable SBSL domain is occupied by bubbles which do not emit light, while above it diffusively unstable SL bubbles are found. The inset shows, at given $P_{a}=1.3 \mathrm{~atm}$, the range of gas concentrations for which stable SBSL is possible (note the logarithmic ordinate).

this approach, an ODE for the gas temperature inside the (uniform) bubble is coupled to the RP equation. Subsequently, the opacity of the heated gas-whose importance was first recognized in the pioneering work of Moss et al. [15] — and the resulting light emission (primarily from thermal bremsstrahlung $[15,16]$ ) are computed, showing good agreement with the available experimental data. Lowering the driving frequency, we predict that, e.g., at $f=5 \mathrm{kHz}$ more than 100 times as many photons are emitted as at $f=20 \mathrm{kHz}$; see Fig. 5a. The maximum temperatures in the bubble reach about $60 \mathrm{kK}$ as compared to $\approx 30 \mathrm{kK}$ at $f=20 \mathrm{kHz}$. The widths of the light pulses can exceed 1000 ps, much longer than hitherto observed [5] for the standard frequencies (Fig. 5b). We make the explicit prediction that for these long and intense light pulses at $5 \mathrm{kHz}$ the width in the red spectral regime should be nearly twice as long as in the blue spectral regime, in contrast to what has been observed at larger $f[5]$.

We suggest to experimentally check the predictions for the light intensities and widths of the light pulses in the proposed parameter regimes, i.e., for low frequencies and the correspondingly low inert gas concentrations (cf. Fig. 4). Such experiments will validate the present understanding of SBSL and may lead to refinements of the model. In particular, one will be able to better judge the role of water vapor. Clearly, the lower $f$, the more water molecules will enter the bubble upon expansion and will modify the light emission process upon collapse [30]. Likewise, the gas composition may become different at the center of the 


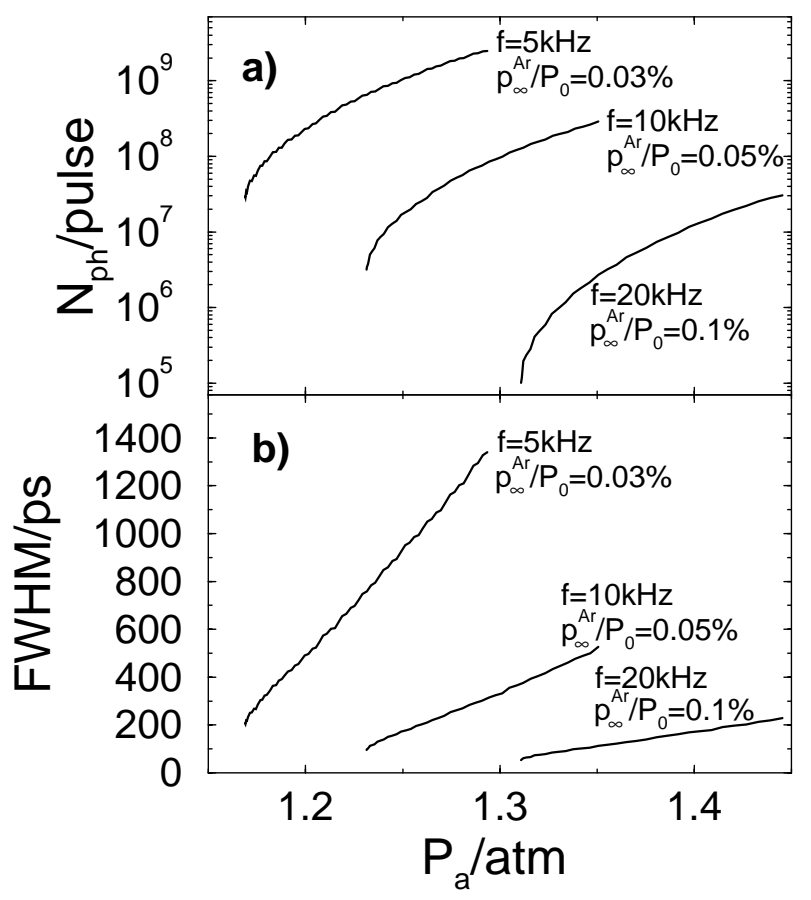

FIG. 5. Number of emitted detectable photons $(200 \mathrm{~nm}<$ $\lambda<800 \mathrm{~nm}$ ) per pulse (a) and pulse width (b) as a function of $P_{a}$ for different driving frequencies. Note that the partial argon pressure has to be decreased for lower $f$.

bubble and near its wall [31]. The neglect of thermal damping [24] will furthermore become more and more questionable for increasing peak temperatures, i.e., for decreasing $f$. Also, the electronic degrees of freedom of argon will eventually limit the compressional heating of the gas.

The good agreement between experiment and theory [16] suggests that the processes mentioned in the previous paragraph are of minor importance in the hitherto explored parameter range of $f=20-40 \mathrm{kHz}$. Therefore, for the time being, we neglected them also in the neighboring regime $f=2-20 \mathrm{kHz}$. Eventually only the comparison to experiment will tell down to which frequencies these approximations are justified and reveal how much further one can push the limits of SBSL towards the region of extreme and extravagant states of matter.

We gratefully acknowledge innumerable discussions with Michael Brenner and Siegfried Grossmann over the years. Without them this work would not have been possible. Support by the Deutsche Forschungsgemeinschaft under Grants No. Lo 556/3-1 and No. SBF185-D8 is also acknowledged.

[1] D.F. Gaitan, Ph.D. thesis, The University of Mississippi, 1990; D.F. Gaitan, L. A. Crum, R.A. Roy, and C.C. Church, J. Acoust. Soc. Am. 91, 3166 (1992).

[2] B. P. Barber et al., Phys. Rep. 281, 65 (1997).
[3] T. Matula et al., Phys. Rev. Lett. 75, 2602 (1995); L. Bernstein, M. Zakin, E. Flint, and K. Suslick, J. Phys. Chem. 100, 6612 (1996).

[4] C. C. Wu and P.H. Roberts, Phys. Rev. Lett. 70, 3424 (1993); L. Frommhold and A. A. Atchley, Phys. Rev. Lett. 73, 2883 (1994); L. Kondic, J. I. Gersten, and C. Yuan, Phys. Rev. E 52, 4976 (1995); W. C. Moss, D. Clarke, J. White, and D. Young, Phys. Lett. A 211, 69 (1995).

[5] B. Gompf et al., Phys. Rev. Lett. 79, 1405 (1997); R. Pecha, B. Gompf, G. Nick, Z.Q. Wang, and W. Eisenmenger, Phys. Rev. Lett. 81, 717 (1998).

[6] R. A. Hiller, S. J. Putterman, and K. R. Weninger, Phys. Rev. Lett. 80, 1090 (1998).

[7] M. Brenner, D. Lohse, and T. Dupont, Phys. Rev. Lett. 75, 954 (1995).

[8] S. Hilgenfeldt, D. Lohse, and M. P. Brenner, Phys. Fluids 8, 2808 (1996).

[9] S. Hilgenfeldt, M.P. Brenner, S. Grossmann, and D. Lohse, J. Fluid Mech. 365, 171 (1998).

[10] D. Lohse et al., Phys. Rev. Lett. 78, 1359 (1997); D. Lohse and S. Hilgenfeldt, J. Chem. Phys. 107, 6986 (1997).

[11] S. Hilgenfeldt, D. Lohse, and W. Moss, Phys. Rev. Lett. 80, 1332 (1998).

[12] R. Apfel (private communication).

[13] B.P. Barber and S. J. Putterman, Nature (London) 352, 318 (1991).

[14] S. Cordry, Ph.D. thesis, The University of Mississippi, 1995.

[15] W. Moss, D. Clarke, and D. Young, Science 276, 1398 (1997).

[16] S. Hilgenfeldt, S. Grossmann, and D. Lohse, Phys. Fluids (to be published).

[17] C. E. Brennen, Cavitation and Bubble Dynamics (Oxford University Press, Oxford, 1995).

[18] M. Plesset, J. Appl. Mech. 16, 277 (1949).

[19] V. Q. Vuong and A. J. Szeri, Phys. Fluids 8, 2354 (1996).

[20] L. Yuan, H. Y. Cheng, M. C. Chu, and P. T. Leung, Phys. Rev. E 57, 4265 (1998); H. Y. Cheng, M. C. Chu, P. T. Leung, and L. Yuan, Phys. Rev. E 58, R2705 (1998).

[21] A. Prosperetti, J. Acoust. Soc. Am 61, 17 (1977); J. Fluid Mech. 222, 587 (1991).

[22] A. Prosperetti, Q. Appl. Math. 34, 339 (1977).

[23] A. Prosperetti, Atti Accad. Naz. Lincei, Rend. Cl. Sci. Fis. Mat. Nat. 62, 196 (1977).

[24] A. Prosperetti and Y. Hao, Philos. Trans. R. Soc. London $\mathbf{x}, \mathrm{xx}(1998)$.

[25] M.P. Brenner, T. Dupont, S. Hilgenfeldt, and D. Lohse, Phys. Rev. Lett. 80, 3668 (1998).

[26] M.P. Brenner, S. Hilgenfeldt, and D. Lohse, in Sonoluminescence and Sonochemistry, edited by L. Crum, NATO ASI (Kluwer Academic Publishers, Dordrecht, The Netherlands, 1998).

[27] A. Eller and H. G. Flynn, J. Acoust. Soc. Am. 37, 493 (1965).

[28] M. M. Fyrillas and A. J. Szeri, J. Fluid Mech. 277, 381 (1994).

[29] G. Holt and F. Gaitan, Phys. Rev. Lett. 77, 3791 (1996).

[30] W. C. Moss et al. (to be published).

[31] B. D. Storey and A. J. Szeri (to be published). 